\title{
In Situ Study on the Evolution of Multimodal Particle Size Distributions of ZnO Quantum Dots: Some General Rules for the Occurrence of Multimodalities
}

\author{
Torben Schindler ${ }^{\mathrm{x}}$, Johannes Walter $\dagger$, Wolfgang Peukert $\dagger$, Doris Segets $\dagger$, Tobias Unruh ${ }^{\mathrm{x}}$
}

${ }^{\mathbf{x}}$ Chair of Crystallography and Structural Physics, Friedrich-Alexander-Universität Erlangen-Nürnberg (FAU), Staudtstr.3, 91058 Erlangen, Germany

$\dagger$ Institute of Particle Technology (LFG), Friedrich-Alexander-Universität Erlangen-Nürnberg (FAU), Cauerstr. 4 , 91058 Erlangen, Germany

\section{SI1: Comparison of analytical methods}

UV/Vis absorption spectroscopy (UV/Vis):

A standard and in situ applicable routine to investigate the dispersity of quantum confined semiconductor NPs with direct band gap is absorbance spectroscopy in the ultraviolet, visible and near infrared regime (UV/Vis/NIR). By this experimental technique the band gap and thus the particle core volume with semiconducting properties of the distributed NPs is accessible. However, for the relation between band gap shift and particle size, the often used effective mass approximation (EMA) overestimates the particle sizes of the small NPs. This was confirmed for ZnO QDs by Viswanatha et al. who compared the EMA results with XRD and TEM measurements. ${ }^{1}$ The same authors introduced a very well accepted and frequently used tight binding model (TBM) that provides the size-dependent band gap energy of ZnO QDs with high accuracy.

In the literature, absorbance spectra are not solely used for the estimation of a mean size but sometimes also for the derivation of size distributions like it has been shown already in 1994 by Mićić et al. for InP QDs. ${ }^{2}$ In 2003, Pesika et al. reported a versatile procedure using the local slope of the absorbance measurement whereas Viswanatha and Sarma described in 2006 a least-square-error approach, where a Gaussian distribution of the PSD is assumed. ${ }^{3,4}$ In this work, an earlier described algorithm developed by Segets et al. is used in combination with the tight binding model of Viswanatha. ${ }^{1,5}$ It allows the calculation of arbitrary shaped multimodal PSDs. Recently, the strategy has been extended to other material systems like $\mathrm{ZnS}$ and PbS(e). ${ }^{6,7}$ For the latter, the authors could use an internal calibration against carefully analyzed TEM micrographs to determine the size dependent band gap energy like it was already proposed in the work of Mićić et al. ${ }^{2}$

Although the applied model for the evaluation of absorbance measurements contains assumptions as e.g. a constant bulk absorption behavior which is shifted to higher energies with decreasing particle size, and is limited to a size range where quantum confinement is observed, clear advantages of the method are identified: it is available in most laboratories, it is non-destructive and can be used in situ at low cost. Having a good correlation of band gap energy and particle size, it provides an excellent positioning of the PSD in terms of the particle diameters that are correlated to volume densities. In combination with a suitable photodiode array the time resolution is on a millisecond scale. ${ }^{8-10}$

Analytical Ultracentrifugation (AUC):

AUC has been proven to be an excellent characterization technique for NPs. ${ }^{11}$ Due to rotor speeds up to 60 $\mathrm{krpm}$ particles smaller than one nanometer are forced to sediment by overcoming the diffusion effects acting on the particles. The radial concentration is measured as a function of time from the axes of rotation to the cell bottom with different detectors. These concentration profiles can be evaluated by several software packages and give access to the sedimentation and diffusion coefficient distributions. ${ }^{12,13}$ This information can be linked to the particulate properties, which is the size, shape and density of the NPs. AUC is an absolute measurement technique and requires no further measurements of reference materials. It is able to resolve PSDs in the range of less than one nanometer to several microns. ${ }^{14-16}$ Even for very small particles multimodal distributions can be derived with Ångström resolution, which makes it superior to other measurement techniques for the analysis of nanoparticles. ${ }^{17}$ Numerous applications within the last years have demonstrated that AUC is a versatile and 
highly resolving method in nanotechnology. ${ }^{14,18-21} \mathrm{ZnO}$ NPs were also investigated in a few studies. Cölfen et al. showed that a sample of medium sized $\mathrm{ZnO}$ NPs $(>4.5 \mathrm{~nm})$ consisted of numerous and discrete size fractions ${ }^{46}$ whereas Wood et al. monitored the evolution of the particle size as a function of the ripening conditions. $^{22}$

Due to the fact that the sedimentation coefficient is dependent upon the mass as well as the hydrodynamic diameter of the particle, the stabilizing ligand shell at the particle surface has to be considered for data evaluation. While the influence of the shell to the measured sedimentation is small for large particles (typically $>10 \mathrm{~nm}$ ), it gets much more important for smaller particles, which have then to be considered as core-shell systems. Hence, the absolute particle density changes with the core size. Knowledge about the size dependent hybrid properties of the particle is required to obtain the correct hydrodynamic and core sizes by AUC. For this, either complementary techniques such as DLS, TEM, UV/Vis or SAXS have to be applied or information on the morphology of the shell has to be deduced from independent methods such as thermal gravimetric analysis (TGA), small angle neutron scattering (SANS), or other techniques. ${ }^{23-26}$ Alternatively, a combined 2-dimensional analysis of the diffusion and sedimentation coefficients can be performed as it was proposed by Carney et al., which will provide the hydrodynamic particle size and density. ${ }^{27}$ The hydrodynamic properties are further influenced by the shape of the particles. Shape anisotropy will increase the hydrodynamic diameter in comparison to a sphere which has the lowest friction factor. Shape has therefore also to be taken into account for the data evaluation.

AUC provides detailed access to the dispersity of samples directly in solution and is superior to most other characterization techniques due to its unrevealed accuracy and resolution. However, in virtue of its measurement concept, it cannot be integrated as an in situ measurement technique in the production process and particles may have to be prevented from further ripening by cooling and high rotor speeds (shorter experiments) due to the comparably long measurement durations of at least one hour. Nevertheless, for the majority of samples it will grant detailed access for PSDs and thus will serve as an excellent complementary technique to the fast UV/Vis and SAXS measurements because designated samples can be investigated with high accuracy allowing the optimization of those in situ methods.

\section{Small Angle X-ray Scattering:}

The scattering signal of an X-ray diffraction (XRD) measurement is directly linked to the electron density distribution within the sample and thus to the concentration, size, and shape of the particles dispersed in a medium. Using SAXS, electron density contrasts on a length scale of the dimensions of NPs are probed and both, the size and the shape of NPs are accessible within a size range from $1 \mathrm{~nm}$ to $1 \mu \mathrm{m}^{28}$ The scattering contrast of most organic and inorganic NPs dispersed in an aqueous or organic medium is sufficiently high to be detected by state-of-the-art SAXS instruments at synchrotron facilities or even conventional X-ray sources. ${ }^{29-34}$

For the interpretation of the SAXS data a profound model is required due to many degrees of freedom (size, shape, polydispersity). A convincing analysis of polydispersity is only possible by cross-validation against additional independent methods. Noteworthy, the SAXS data of this study could also be described with high accuracy by using ellipsoidal or cylindrical NPs with small aspect ratios. Thus, information on particle shape and polydispersity has to be known from literature or provided by complementary techniques.

The combination of SAXS with absorption spectroscopy was used by Caetano et al. to monitor the agglomeration of $\mathrm{ZnO} \mathrm{NPs},{ }^{35}$ however without reporting on the appearance of additional small particles. In our study a different cation and a different $\mathrm{Zn}^{2+}: \mathrm{OH}^{-}$ratio is used. Both might have a significant effect on $\mathrm{ZnO}$ nucleation, growth, and ripening/aggregation. ${ }^{36-38}$ Briois et al. tested the influence of water on the ripening of ZnO NPs using UV/Vis and SAXS and determined a high polydispersity. ${ }^{39}$ However, they could not probe the PSD using SAXS directly as their $Q$-resolution was limited due to the applied geometry. To circumvent these problems we used a wider $Q$-range for our study to achieve a full view of the PSD evolution.

Thus, SAXS is an excellent in situ applicable technique that can be combined with stopped-flow approaches and is applicable to a wide particle size range. However, it is comparatively expensive and requires a careful evaluation of the applied models. 


\section{SI2: Photograph of SAXS instrument}

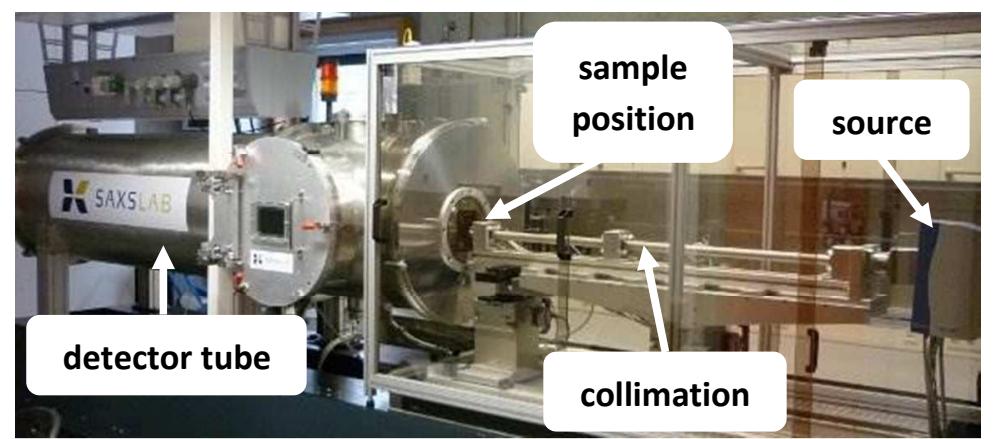

Figure S2: Photograph of the unique and highly customized SAXS instrument which was used for the SAXS measurements can be seen above. The instrument has been developed by SAXSLab (Skovlunde, Denmark) in close cooperation with our group. On the right hand side the microfocus $30 \mathrm{~W}$ Genix X-ray source with a low divergence optics (Xenocs, Grenoble) provides a collimated $\mathrm{Cu}-\mathrm{K}_{\alpha}$ X-ray beam $(\lambda=0.154 \mathrm{~nm})$ with a flux of about $3 \times 10^{8}$ photons per second. The X-rays are collimated in by two sets of double slit systems which are adjusted for a beam size at the sample position of $0.4 \times 0.4 \mathrm{~mm}^{2}$. The sample position used for the presented studies is at ambient conditions and located about $50 \mathrm{~mm}$ behind the second slit and $30 \mathrm{~mm}$ in front of the $2.5 \mathrm{~m}$ long detector tube as indicated in the image. The whole X-ray path of the instrument is evacuated except a length of about $50 \mathrm{~mm}$ around the sample. The water-cooled and vacuum tight Pilatus $3300 \mathrm{~K}$ detector (Dectris AG, Baden, Switzerland) is located inside the detector tube and can be moved by a high-precision 3D positioning system which allows to vary the sample-detector-distances (SDDs) between $100 \mathrm{~mm}$ and $2100 \mathrm{~mm}$. Mica sheets of $10 \mu \mathrm{m}$ thickness are used as beam windows before and behind the sample.

\section{SI3: SAXS PSD with single fractions}

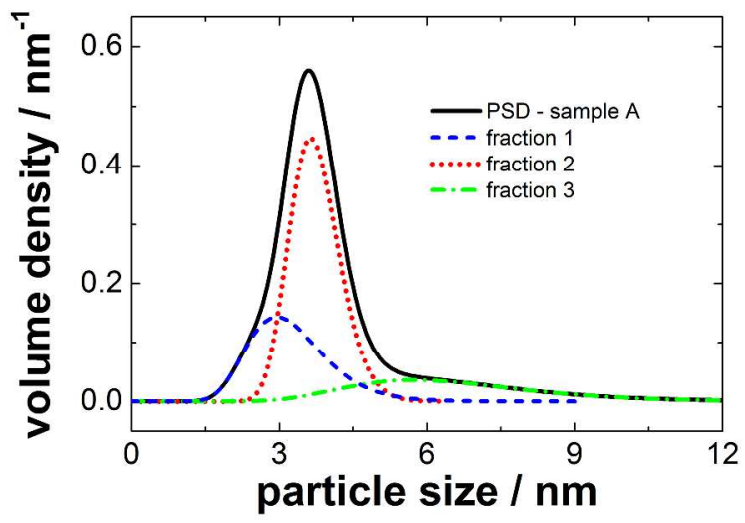

Figure S3. PSD of sample A as determined from the SAXS fit (black line). The single fractions are shown in blue (small-sized particle fraction), red (medium-sized particle fraction) and green (large-sized particle fraction). 


\section{SI4: PSD of a Monte Carlo SAXS analysis}
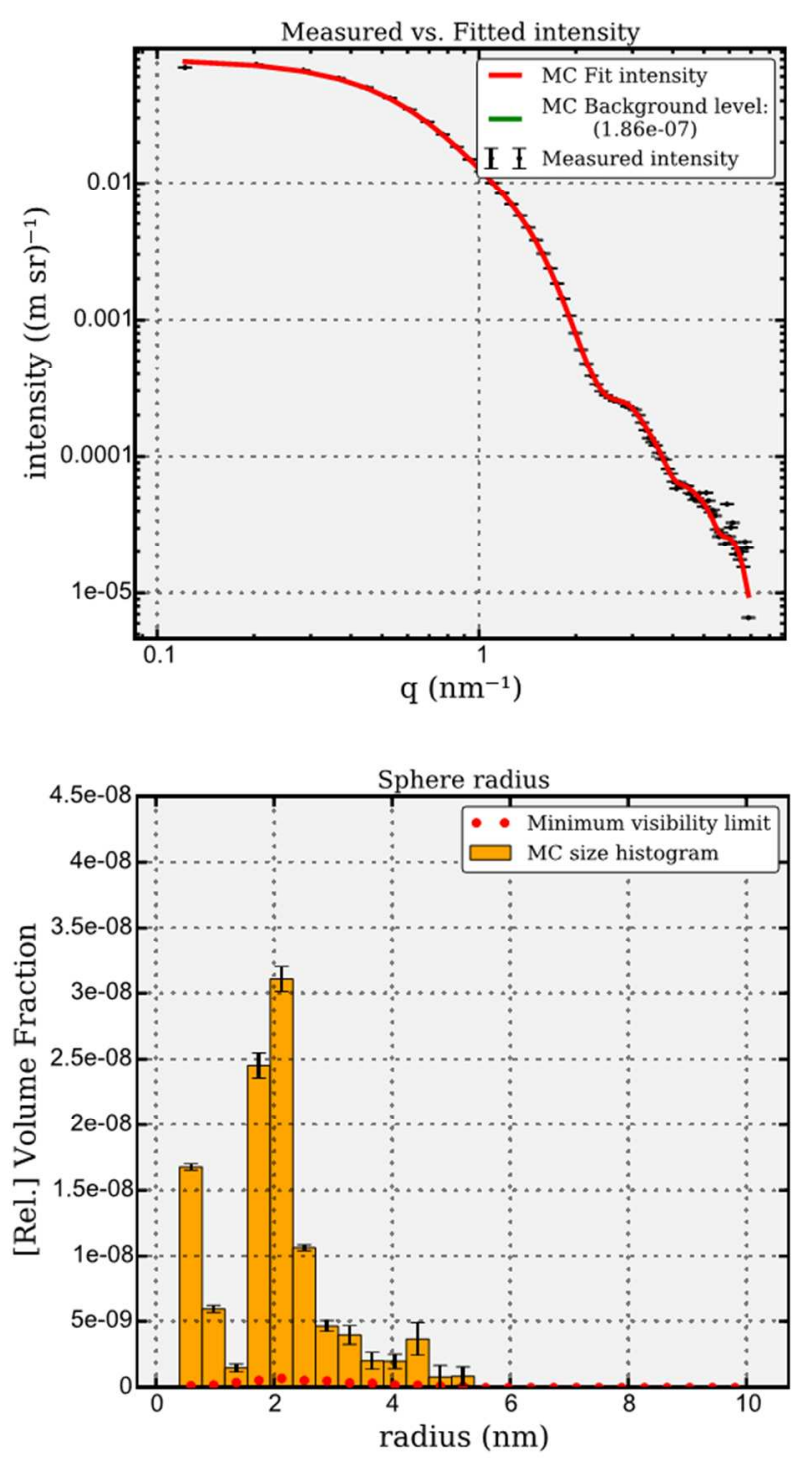

Figure S4. Monte Carlo data analysis for sample D $(360 \mathrm{~min})$. The upper plot shows the data and the Monte Carlo fit result, while the lower picture shows the underlying particle size distributions. It can be clearly seen that beneath the main fraction of particles with radii of about $r=2.3 \mathrm{~nm}$, a fraction of small particles $\sim 1 \mathrm{~nm}$ exists. In addition a third maximum was detected at $\mathrm{r}=4.5 \mathrm{~nm}$ indicating the fraction of larger agglomerates. 


\section{SI5-7: Further information for the AUC analysis}

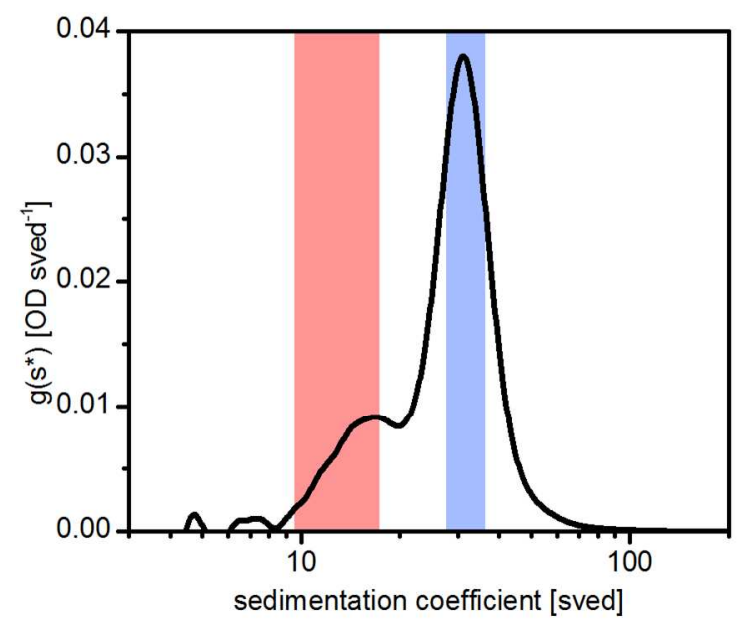

Figure S5. Apparent non-diffusion correct sedimentation coefficient distribution of ZnO NPs after $360 \mathrm{~min}$ (sample D) as obtained by wide distribution analysis (WDA) in SedAnal. ${ }^{16,40}$ Colored bars give the ranges for the small (red) and large fraction (blue) applied for extraction of absorbance spectra using multiwavelength analysis. $^{41}$

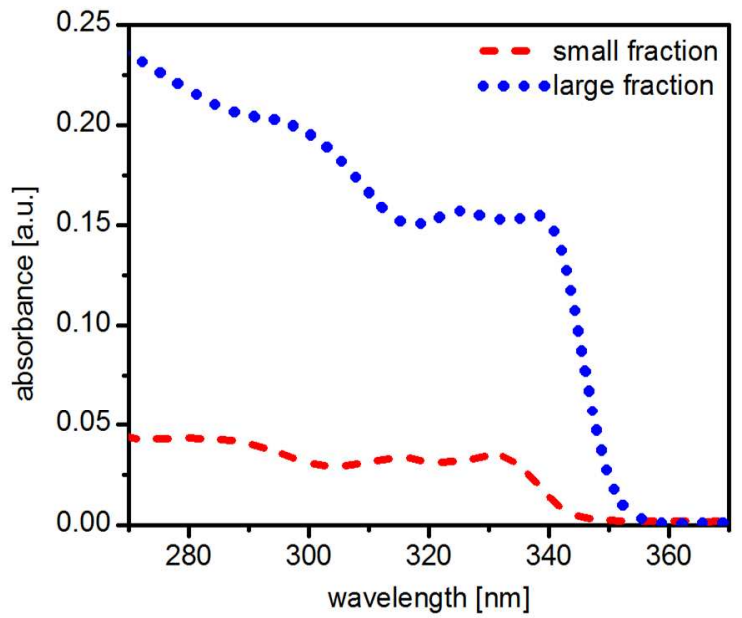

Figure S6. Absorbance spectra of ZnO NPs after $360 \mathrm{~min}$ (sample) as obtained for the small (black) and large fraction (red) using multiwavelength analysis and ranges provided in Figure S5. 


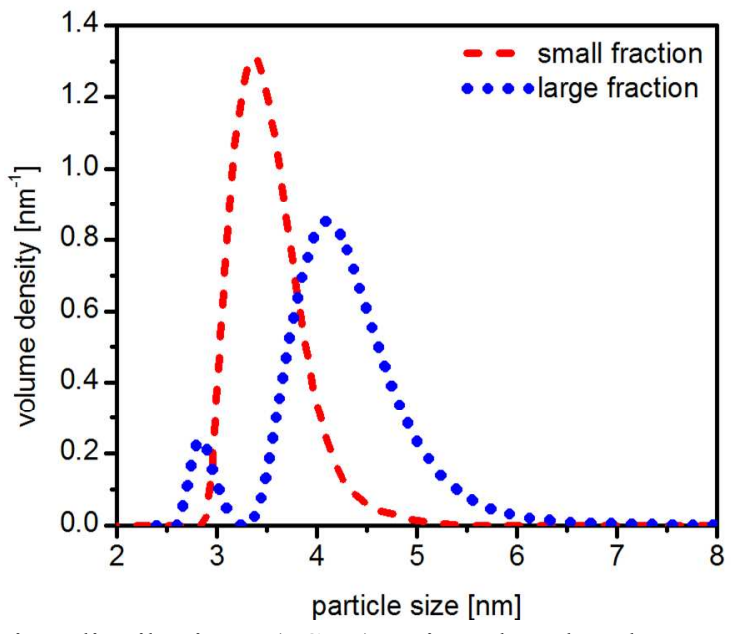

Figure S7. Derived particle size distributions (PSDs) using the absorbance deconvolution for both spectra shown in Figure S6. Shift in mean core diameter for large fraction is clearly observable. The small peak below $3 \mathrm{~nm}$ found for the large fraction is presumably due to spectral artefact in the extracted spectrum and should therefore not be considered for further comparison with hydrodynamic data obtained by AUC. 


\section{SI8: Evolution of $\mathrm{ZnO}$ volume at $20^{\circ} \mathrm{C}$}

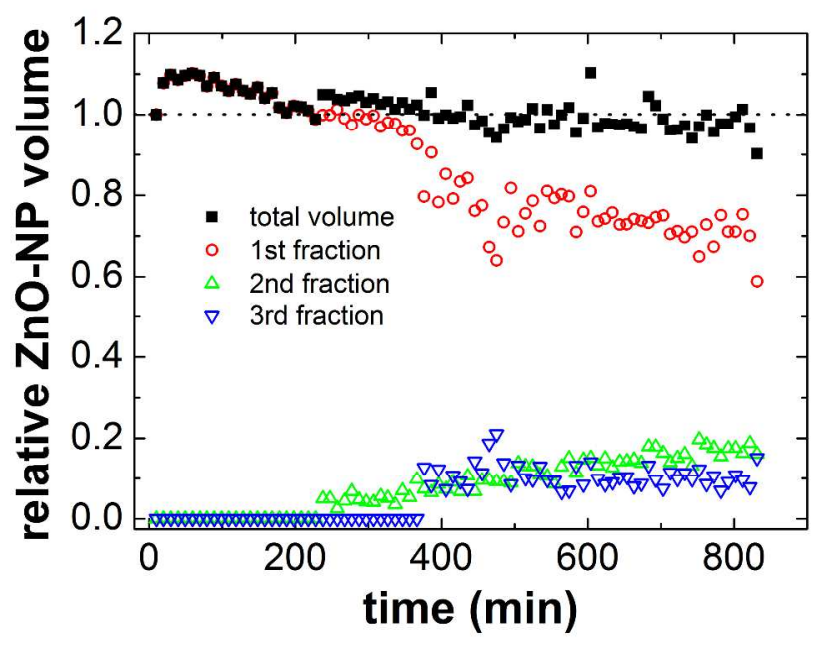

Figure S8. Scheme of the evolution of the $\mathrm{ZnO}$ volume within the different particle fractions for ageing at $20{ }^{\circ} \mathrm{C}$.

\section{References}

1. Viswanatha, R.; Sapra, S.; Satpati, B.; Satyam, P. V.; Dev, B. N.; Sarma, D. D., Understanding the quantum size effects in ZnO nanocrystals. Journal of Materials Chemistry 2004, 14, 661-668.

2. Micic, O. I.; Curtis, C. J.; Jones, K. M.; Sprague, J. R.; Nozik, A. J., Synthesis and Characterization of InP Quantum Dots. The Journal of Physical Chemistry 1994, 98, 4966-4969.

3. Pesika, N. S.; Stebe, K. J.; Searson, P. C., Determination of the Particle Size Distribution of Quantum Nanocrystals from Absorbance Spectra. Advanced Materials 2003, 15, 1289-1291.

4. Viswanatha, R.; Sarma, D. D., Study of the Growth of Capped ZnO Nanocrystals: A Route to Rational Synthesis. Chemistry - A European Journal 2006, 12, 180-186.

5. Segets, D.; Martinez Tomalino, L.; Gradl, J.; Peukert, W., Real-Time Monitoring of the Nucleation and Growth of ZnO Nanoparticles Using an Optical Hyper-Rayleigh Scattering Method. The Journal of Physical Chemistry C 2009, 113, 11995-12001.

6. Segets, D.; Komada, S.; Butz, B.; Spiecker, E.; Mori, Y.; Peukert, W., Quantitative evaluation of size selective precipitation of Mn-doped ZnS quantum dots by size distributions calculated from UV/Vis absorbance spectra. J Nanopart Res 2013, 15, 1-13.

7. $\quad$ Segets, D.; Lucas, J. M.; Klupp Taylor, R. N.; Scheele, M.; Zheng, H.; Alivisatos, A. P.; Peukert, W., Determination of the Quantum Dot Band Gap Dependence on Particle Size from Optical Absorbance and Transmission Electron Microscopy Measurements. ACS Nano 2012, 6, 9021-9032.

8. Tiemann, M.; Weiß, Ö.; Hartikainen, J.; Marlow, F.; Lindén, M., Early Stages of ZnS Nanoparticle Growth Studied by In-Situ Stopped-Flow UV Absorption Spectroscopy. ChemPhysChem 2005, 6, 21132119. 
9. Mori, J.; Miyashita, Y.; Oliveira, D.; Kasai, H.; Oikawa, H.; Nakanishi, H., Stopped-flow analysis on the mechanism of perylene nanoparticle formation by the reprecipitation method. Journal of Crystal Growth 2009, 311, 553-555.

10. Brazeau, A. L.; Jones, N. D., Growth Mechanisms in Nanocrystalline Lead Sulfide by Stopped-Flow Kinetic Analysis. The Journal of Physical Chemistry C 2009, 113, 20246-20251.

11. Mächtle, W.; Börger, L., Analytical Ultracentrifugation of Polymers and Nanoparticles. SpringerVerlag: Berlin, Heidelberg, 2006.

12. Brookes, E.; Cao, W.; Demeler, B., A Two-Dimensional Spectrum Analysis for Sedimentation Velocity Experiments of Mixtures with Heterogeneity in Molecular Weight and Shape. European Biophysics Journal 2010, 39, 405-14.

13. Schuck, P., Size-distribution Analysis of Macromolecules by Sedimentation Velocity Ultracentrifugation and Lamm Equation Modeling. Biophysical Journal 2000, 78, 1606-1619.

14. Planken, K. L.; Colfen, H., Analytical Ultracentrifugation of Colloids. Nanoscale 2010, 2, 18491869.

15. Scott, D. J.; Harding, S. E.; Rowe, A. J., Analytical Ultracentrifugation: Techniques and Methods. Royal Society of Chemistry: 2005.

16. Stafford, W. F.; Braswell, E. H., Sedimentation Velocity, Multi-speed Method for Analyzing Polydisperse Solutions. Biophysical Chemistry 2004, 108, 273-279.

17. Cölfen, H.; Pauck, T., Determination of Particle Size Distributions With Angström Resolution. Colloid and Polymer Science 1997, 275, 175-180.

18. Fagan, J. A.; Zheng, M.; Rastogi, V.; Simpson, J. R.; Khripin, C. Y.; Silvera Batista, C. A.; Hight Walker, A. R., Analyzing Surfactant Structures on Length and Chirality Resolved $(6,5)$ Single-Wall Carbon Nanotubes by Analytical Ultracentrifugation. ACS Nano 2013, 7, 3373-3387.

19. Karabudak, E.; Wohlleben, W.; Cölfen, H., Investigation of Beta-Carotene-Gelatin Composite Particles with a Multiwavelength UV/vis Detector for the Analytical Ultracentrifuge. European Biophysics Journal 2010, 39, 397-403.

20. Zook, J. M.; Rastogi, V.; MacCuspie, R. I.; Keene, A. M.; Fagan, J., Measuring Agglomerate Size Distribution and Dependence of Localized Surface Plasmon Resonance Absorbance on Gold Nanoparticle Agglomerate Size Using Analytical Ultracentrifugation. ACS Nano 2011, 5, 8070-8079.

21. Walter, J.; Löhr, K.; Karabudak, E.; Reis, W.; Mikhael, J.; Peukert, W.; Wohlleben, W.; Cölfen, H., Multidimensional Analysis of Nanoparticles with Highly Disperse Properties Using Multiwavelength Analytical Ultracentrifugation. ACS Nano 2014, 8, 8871-8886.

22. Wood, A.; Giersig, M.; Hilgendorff, M.; Vilas-Campos, A.; Liz-Marz; \#225; n, L. M.; Mulvaney, P., Size Effects in ZnO: The Cluster to Quantum Dot Transition. Australian Journal of Chemistry 2003, 56, 1051-1057.

23. Lees, E. E.; Gunzburg, M. J.; Nguyen, T.-L.; Howlett, G. J.; Rothacker, J.; Nice, E. C.; Clayton, A. H. A.; Mulvaney, P., Experimental Determination of Quantum Dot Size Distributions, Ligand Packing Densities, and Bioconjugation Using Analytical Ultracentrifugation. Nano Letters 2008, 8, 2883-2890.

24. Benoit, D. N.; Zhu, H.; Lilierose, M. H.; Verm, R. A.; Ali, N.; Morrison, A. N.; Fortner, J. D.; Avendano, C.; Colvin, V. L., Measuring the Grafting Density of Nanoparticles in Solution by Analytical Ultracentrifugation and Total Organic Carbon Analysis. Analytical Chemistry 2012, 84, 9238-9245.

25. Nontapot, K.; Rastogi, V.; Fagan, J. A.; Reipa, V., Size and density measurement of core-shell Si nanoparticles by analytical ultracentrifugation. Nanotechnology 2013, 24, 0957-4484.

26. Marczak, R.; Segets, D.; Voigt, M.; Peukert, W., Optimum between purification and colloidal stability of ZnO nanoparticles. Adv. Powder Technol. 2010, 21, 41-49.

27. Carney, R. P.; Kim, J. Y.; Qian, H.; Jin, R.; Mehenni, H.; Stellacci, F.; Bakr, O. M., Determination of nanoparticle size distribution together with density or molecular weight by 2D analytical ultracentrifugation. Nat. Commun. 2011, 2, 335. 
28. Keshari, A. K.; Pandey, A. C., Size and Distribution: A Comparison of XRD, SAXS and SANS Study of II-VI Semiconductor Nanocrystals. Journal of Nanoscience and Nanotechnology 2008, 8, 1221-1227.

29. Bunjes, H.; Unruh, T., Characterization of lipid nanoparticles by differential scanning calorimetry, X-ray and neutron scattering. Advanced Drug Delivery Reviews 2007, 59, 379-402.

30. Schmiele, M.; Schindler, T.; Unruh, T.; Busch, S.; Morhenn, H.; Westermann, M.; Steiniger, F.; Radulescu, A.; Lindner, P.; Schweins, R.; Boesecke, P., Structural characterization of the phospholipid stabilizer layer at the solid-liquid interface of dispersed triglyceride nanocrystals with small-angle $\mathrm{x}$-ray and neutron scattering. Phys. Rev. E 2013, 87, 062316.

31. Abécassis, B.; Testard, F.; Spalla, O.; Barboux, P., Probing in situ the Nucleation and Growth of Gold Nanoparticles by Small-Angle X-ray Scattering. Nano Letters 2007, 7, 1723-1727.

32. Harada, M.; Katagiri, E., Mechanism of Silver Particle Formation during Photoreduction Using In Situ Time-Resolved SAXS Analysis. Langmuir 2010, 26, 17896-17905.

33. Tokumoto, M. S.; Pulcinelli, S. H.; Santilli, C. V.; Craievich, A. F., SAXS study of the kinetics of formation of ZnO colloidal suspensions. J. Non-Cryst. Solids 1999, 247, 176-182.

34. Schmiele, M.; Gehrer, S.; Westermann, M.; Steiniger, F.; Unruh, T., Formation of liquid crystalline phases in aqueous suspensions of platelet-like tripalmitin nanoparticles. The Journal of Chemical Physics 2014, 140, -.

35. Caetano, B. L.; Santilli, C. V.; Meneau, F.; Briois, V. r.; Pulcinelli, S. H., In Situ and Simultaneous UV-vis/SAXS and UV-vis/XAFS Time-Resolved Monitoring of ZnO Quantum Dots Formation and Growth. J. Phys. Chem. C 2011, 115, 4404-4412.

36. Santra, P. K.; Mukherjee, S.; Sarma, D. D., Growth Kinetics of ZnO Nanocrystals in the Presence of a Base: Effect of the Size of the Alkali Cation. The Journal of Physical Chemistry C 2010, 114, 2211322118.

37. Viswanatha, R.; Amenitsch, H.; Sarma, D. D., Growth Kinetics of ZnO Nanocrystals: A Few Surprises. Journal of the American Chemical Society 2007, 129, 4470-4475.

38. Viswanatha, R.; Santra, P. K.; Dasgupta, C.; Sarma, D. D., Growth Mechanism of Nanocrystals in Solution: ZnO, a Case Study. Physical Review Letters 2007, 98, 255501.

39. Briois, V.; Giorgetti, C.; Dartyge, E.; Baudelet, F.; Tokumoto, M.; Pulcinelli, S.; Santilli, C., In situ and simultaneous nanostructural and spectroscopic studies of $\mathrm{ZnO}$ nanoparticle and $\mathrm{Zn}$-HDS formations from hydrolysis of ethanolic zinc acetate solutions induced by water. Journal of Sol-Gel Science and Technology 2006, 39, 25-36.

40. Stafford, W. F., Boundary analysis in sedimentation transport experiments: a procedure for obtaining sedimentation coefficient distributions using the time derivative of the concentration profile. Analytical Biochemistry 1992, 203, 295-301.

41. Walter, J.; Sherwood, P. J.; Lin, W.; Segets, D.; Stafford, W. F.; Peukert, W., Simultaneous Analysis of Hydrodynamic and Optical Properties Using Analytical Ultracentrifugation Equipped with Multiwavelength Detection. Analytical Chemistry 2015, 87, 3396-3403. 